\title{
Le gainage précoce des régimes de bananes améliore la croissance des fruits et leur état sanitaire vis-à-vis de l'anthracnose (Colletotrichum musae)
}

Joseph Mouen Bedimo ${ }^{a}$, Marc Chillet ${ }^{b}$, Alexandra Jullien $^{b}$, Luc de Lapeyre de Bellaire ${ }^{b *}$

a Irad, Station de Foumbot, BP 665 Bafoussam,

Cameroun

${ }^{b}$ Cirad-flhor,

Station de Neufchâteau, 97130 Guadeloupe,

France

lapeyre@cirad.fr
* Correspondance et tirés à part

Reçu le 4 juin 2002 Accepté le 16 octobre 2002

Fruits, 2003, vol. 58, p. 71-81 (C) 2003 Cirad/EDP Sciences All rights reserved DOI: $10.1051 /$ fruits:2002037

RESUMEN ESPAÑOL, p. 81
The early bunch covering of banana improves the growth of fruit and its sanitary state with respect to anthracnosis (Colletotrichum musae).

Abstract - Introduction. Banana anthracnosis, caused by Colletotrichum musae, is a storage disease which is generally controlled by fungicidal treatments after harvest. Other means of control are today required to replace this chemical control, such as deflowering and bunch covering, usually carried out at the "horizontal fingers" stage (Hfing). Taking into account the precocity of the fruit pollution by C. musae of the part played by rainwater as agent of dissemination of the conidia, and the barrier effect which the bunch cover plays in this dissemination, we wanted to assess the consequences, on the level of fruit contamination, of an early or late bunch covering. In addition, we wanted to evaluate the possibility of replacing the deflowering in the field by a bunch covering at a very early stage easier to carry out. Lastly, we wanted to check that an early bunch cover could involve better filling of fruit. Materials and methods. We compared various covering stages (Hfing-7 d, Hfing, Hfing+7 d, Hfing+14 d, bunch not covered) with respect to their effects on the fruit contamination, but also on its growth (fruit cell number, average fruit weight). Early covering (Hfing-7 d) was also compared with the combination deflowering/covering at the Hfing stage, which is an effective technique, but expensive in labour. Results. The fruit contamination rates were significantly higher on late covered bunches (Hfing $+7 \mathrm{~d}$, Hfing $+14 \mathrm{~d}$ ) or, worse, on not covered bunches. The lowest contamination rates were measured on the early covered bunch fruit (Hfing-7 d) or in Hfing, without significant differences between these two treatments. In addition, early covering did not reduce the fruit contamination as much as the deflowering in the field associated with the bunch covering at the Hfing stage. Lastly, the bunch covering stage influenced the growth of the fruits. Conclusion. Early bunch covering before the Hfing stage, or even at an earlier stage (Hfing-7), is recommended.

France (Guadeloupe) / Musa acuminata / cultural control / anthracnosis / Colletotrichum musae / contamination / plant developmental stages / growth / bunch covering

\section{Le gainage précoce des régimes de bananes améliore la croissance des} fruits et leur état sanitaire vis-à-vis de l'anthracnose (Colletotrichum musae).

Résumé - Introduction. L'anthracnose des bananes, causée par Colletotrichum musae est une maladie de conservation qui est généralement contrôlée par des traitements fongicides après la récolte. D'autres moyens de lutte sont aujourd'hui recherchés pour remplacer cette lutte chimique, dont l'épistillage et le gainage des inflorescences effectué habituellement au stade " doigts horizontaux " (Sdh). Compte tenu de la précocité de la pollution des fruits par C. musae, du rôle joué par l'eau de pluie comme agent de dissémination des conidies et de l'effet barrière que joue la gaine dans cette dissémination, nous avons voulu évaluer les conséquences, sur le niveau de contamination des fruits, d'un gainage précoce ou tardif. Par ailleurs, nous avons voulu évaluer la possibilité de remplacer l'épistillage au champ par un gainage à un stade très précoce plus facile à effectuer. Enfin, nous avons voulu vérifier qu'un gainage précoce pouvait entraîner un meilleur remplissage des fruits. Matériel et méthodes. Nous avons comparé différents stades de gainage (Sdh-7 j, Sdh, Sdh+7 j, Sdh+14 j, régimes non gainés) vis-à-vis de leurs effets sur la contamination des fruits, mais aussi sur leur croissance (nombre de cellules par fruit, poids moyen du fruit). Le gainage précoce (Sdh-7) a aussi été comparé à la combinaison épistillage/gainage au stade Sdh qui est une technique efficace mais coûteuse en main d'œuvre. Résultats. Les niveaux de contamination des fruits ont été significativement les plus élevés sur des régimes gainés tardivement $(\mathrm{Sdh}+7, \mathrm{Sdh}+14)$ ou, pires, sur des régimes non gainés. Les taux de contamination les plus faibles ont été mesurés sur les fruits gainés précocement (Sdh-7) ou au Sdh, sans qu'il n'y ait de différences significatives entre ces deux traitements. Par ailleurs, le gainage précoce n'a pas autant réduit la contamination des fruits que l'épistillage au champ associé au gainage Sdh. Enfin, le stade de gainage a influencé la croissance des fruits. Conclusion. La pose précoce de la gaine avant le stade Sdh, voire à un stade plus précoce (Sdh-7), est recommandée.

France (Guadeloupe) / Musa acuminata / lutte culturale / anthracnose / Colletotrichum musae / contamination / stade de développement végétal / croissance / gainage 


\section{Introduction}

Aux Antilles françaises, la qualité des bananes d'exportation est altérée par le développement des maladies de conservation et par des problèmes de "mûrs d'arrivage ". En effet, du fait d'un métabolisme très actif, les bananes disposent d'un faible potentiel de conservation qui se traduit parfois par un mûrissage précoce pendant leur transport vers l'Europe. De telles bananes subissent alors une décote lors de leur commercialisation. Si ces problèmes de "mûrs d'arrivage " sont globalement maîtrisés par le contrôle de la date de coupe $[1,2]$ et par la réfrigération des fruits à $13{ }^{\circ} \mathrm{C}$ pendant leur transport, il en va autrement pour les maladies de conservation qui nécessitent une stratégie de lutte plus complexe. C'est le cas de l'anthracnose, maladie causée par Colletotrichum musae et qui est l'un des facteurs les plus pénalisants de la qualité des bananes d'exportation antillaises. Le parasite contamine les fruits au champ et forme rapidement des appressoria qui restent dans un état quiescent jusqu'à la maturation du fruit [3, 4]. Ces appressoria vont assurer la fixation du parasite sur l'hôte, sa survie dans des conditions très adverses, et aussi assurer la pénétration dans les tissus de l'hôte [5].

L'anthracnose se manifeste par des tâches nécrotiques sombres, en dépression et aux contours plus ou moins nets sur le péricarpe des fruits. En vieillissant, ces tâches s'agrandissent, deviennent coalescentes et s'étendent sur l'ensemble du fruit, occasionnant ainsi sa pourriture jusqu'au niveau de la pulpe. En fonction du mode de pénétration du parasite, Meredith [6] a distingué deux formes de la maladie. La première, l'anthracnose de blessure, survient en cas de blessure du péricarpe. Dans ce cas, les lésions sont très larges et apparaissent généralement très rapidement durant le transport réfrigéré des fruits. La deuxième, l'anthracnose de quiescence, se traduit par l'apparition plus tardive, en sortie de mûrisserie ou chez les distributeurs, de lésions de plus petite taille. Bien que les fruits soient contaminés au champ, les symptômes de la maladie ne sont visibles qu'au cours de la mise en marché des fruits, ce qui ne facilite pas une prise de conscience rapide du problème par les producteurs de bananes.

Le recours à des fongicides systémiques en traitement après récolte constitue le moyen couramment utilisé pour contrôler cette maladie [7]. Toutefois, cette lutte chimique est remise en cause par l'apparition de souches résistantes qui résulteraient de l'emploi de fongicides ayant le même mode d'action que ceux utilisés en traitement aérien contre la cercosporiose du bananier [8].

Par ailleurs, la culture industrielle de la banane aux Antilles est fortement mise en cause dans les problèmes de dégradation de l'environnement. Cette culture implique en effet l'usage de nombreux pesticides dont certains, très toxiques comme les nématicides, sont responsables de la pollution de cours d'eau [9]. La pression de l'opinion publique vis-à-vis de la pollution de l'environnement contribue à ternir davantage l'image de cette banane qui est déjà fortement affaiblie sur le marché international. Enfin, il y a aujourd'hui des exigences très fortes des consommateurs en matière de sécurité alimentaire et donc une demande soutenue pour des produits ne faisant pas l'objet de traitements après récolte. Ainsi, des solutions autres que l'emploi de fongicides après la récolte permettraient de conférer à la production des Antilles françaises un signe distinctif de qualité qui pourrait être valorisé sur de nouveaux segments commerciaux.

Dans les conditions tropicales humides de la Guadeloupe, les niveaux de contamination des fruits sont très élevés dans les parcelles mal entretenues et exposées à de fortes pluviométries [10]. Des travaux antérieurs ont montré que la pollution des fruits s'exerce majoritairement au cours des 40 premiers jours qui suivent la sortie de l'inflorescence, et que les pièces florales et la bractée inférieure du régime sont les principales sources d'inoculums efficaces pour la contamination des fruits [11, 12]. Par ailleurs, il a été montré que le ruissellement de l'eau de pluie est un facteur indispensable au transport de l'inoculum à la surface des fruits [12]. Ainsi, certaines 
pratiques culturales comme l'épistillage au champ (élimination des sources d'inoculum), et le gainage des régimes (entrave au ruissellement de l'eau de pluie) peuvent contribuer à limiter sensiblement le niveau de contamination des fruits et donc à s'affranchir des traitements après récolte.

La gaine est ordinairement posée au stade " doigts horizontaux ", soit généralement (10 à 13) j après la sortie de l'inflorescence. Compte tenu de la précocité de la pollution des fruits, du rôle joué par l'eau de pluie comme agent de dissémination des conidies et de l'effet barrière que joue la gaine dans cette dissémination, nous avons voulu évaluer les conséquences, sur le niveau de contamination des fruits, d'un gainage précoce - donc avant l'anthèse, à (3 à 5) $\mathrm{j}$ après la sortie de l'inflorescence ou tardif - soit (7 et 14) j après le stade " doigts horizontaux". L'objectif de ces expérimentations a alors été de préciser les recommandations faites aux producteurs.

Par ailleurs, nous avons voulu évaluer la possibilité de remplacer l'épistillage au champ, qui est une opération efficace mais très contraignante et coûteuse en main d'œuvre, par une technique moins lourde à effectuer. La technique testée a alors été le gainage à un stade très précoce, juste avant l'anthèse de la première bractée.

Enfin, un modèle de remplissage des fruits élaboré récemment [2] laisserait entrevoir qu'un gainage précoce pourrait entrâ̂ner un meilleur remplissage des fruits. Nous avons donc voulu vérifier cette hypothèse.

\section{Matériel et méthodes}

\subsection{Matériel végétal}

Les expérimentations ont été conduites en bananeraies constituées de plants appartenant au cultivar 'Grande naine' (Musa acuminata, triploïde AAA, sous-groupe Cavendish).

\subsection{La gaine et les différents stades de gainage}

La gaine utilisée est un sac en polyéthylène de couleur bleue, de (1,50 à 1,70) $\mathrm{m}$ de longueur et $20 \mu \mathrm{m}$ d'épaisseur, présentant plusieurs petites perforations de $8 \mathrm{~mm}$ de diamètre qui permettent l'aération des fruits. Les stades de gainage ont été définis sur un pas de temps hebdomadaire, en fonction du stade de développement floral et de la dynamique de contamination des fruits (figure 1).

- Le gainage précoce, identifié $\mathrm{Sdh}_{-7}$, a été effectué 7 jours avant le stade "doigts horizontaux". Se situant avant l'anthèse de la première main, soit environ 5 jours après la jetée de l'inflorescence, c'est le stade le plus précoce auquel il est possible de poser une gaine sur un régime de bananes car, pour cette opération, l'inflorescence doit être suffisamment développée pour que la première bractée soit supprimée et que la gaine soit alors posée " en cloche" au-dessus de la cicatrice d'insertion de la bractée.

- Le gainage au stade "doigts horizontaux" (Sdh) est celui qui est actuellement recommandé pour la pose de la gaine. À ce stade de développement floral, l'inoculum n'est pas encore abondant sur les pièces florales.

- Le gainage effectué 7 jours après le stade "doigts horizontaux" $\left(\mathrm{Sdh}_{+7}\right)$ est

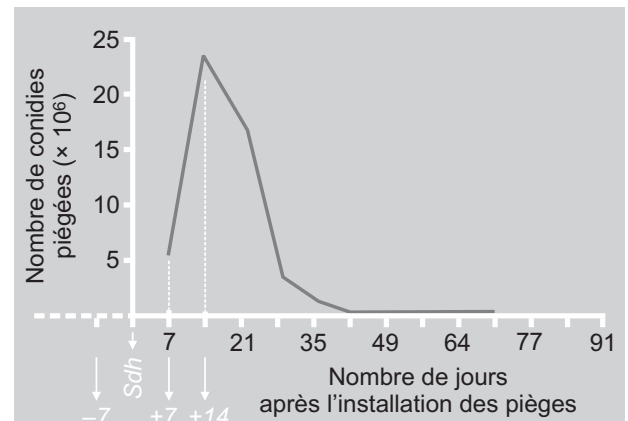

Figure 1.

Définition de différents stades de pose d'une gaine sur des inflorescences de bananiers en fonction de la dynamique de piégeage des spores mesurée à partir du stade " doigts horizontaux ». 
parfois réalisé lorsqu'il y a une surcharge de travail dans les exploitations agricoles. À ce stade d'évolution des fruits, l'inoculum devient abondant sur les pièces florales.

- Le gainage peut être parfois réalisé deux semaines après le stade "doigts horizontaux " $\left(\mathrm{Sdh}_{+14}\right)$ lorsqu'il y a des contraintes de calendrier de travaux. La production d'inoculum sur les pièces florales est alors maximale.

\subsection{Marquage des bananiers}

Le marquage des bananiers a consisté à repérer des plants ayant un même stade visible de développement, dès la sortie de l'inflorescence, et à les répartir en fonction des traitements expérimentaux. Cette opération a été effectuée de façon échelonnée du fait de l'hétérogénéité des dates de floraison des plants au sein d'une même parcelle.

Chaque semaine de floraison a été considérée comme une répétition d'un même traitement. Chaque semaine, les plants repérés ont été répartis aléatoirement entre les différents traitements correspondant aux stades de gainage afin de minimiser les biais dus à une éventuelle hétérogénéité du sol et du microclimat à divers points de la parcelle d'essai. Ainsi, les bananiers de même âge physiologique ont pu être identifiés par un ruban de couleur spécifique attaché autour du pseudo-tronc. Chaque traitement (stade de gainage) a été caractérisé par une peinture de couleur spécifique sur le pseudo-tronc. Par ailleurs, tous les bananiers expérimentaux ont été identifiés par un numéro individuel inscrit sur une étiquette de même couleur que celle du traitement.

\subsection{Influence du stade de gainage sur la contamination des fruits}

Une expérimentation testant l'influence du stade de gainage sur la contamination des fruits a été menée dans une bananeraie de deuxième cycle située à $200 \mathrm{~m}$ d'altitude de la côte au vent, un site où la pression infectieuse observée est élevée. Cette par- celle avait déjà fait l'objet d'études antérieures [12]. Des inflorescences ayant été gainées aux quatre stades préalablement définis $\left(\mathrm{Sdh}_{-7}, \mathrm{Sdh}, \mathrm{Sdh}_{+7}\right.$ et $\mathrm{Sdh}_{+14}$ ) et un témoin non gainé et non épistillé ont été comparés entre eux. De juin à juillet 2000, six répétitions ont été réalisées au cours de six semaines consécutives de marquage. Chaque semaine, 200 bananiers ont été marqués ; chaque traitement a ainsi été représenté par un ensemble de 40 bananiers complètement dispersés dans le champ d'essai.

Les niveaux de contamination ont été mesurés sur trois fruits prélevés respectivement sur la première, la médiane et la dernière main de chaque régime expérimental. Les observations ont été réalisées à sept semaines après la date de la sortie de l'inflorescence et au stade de récolte fixé à 900 degrés jour après le stade Sdh [1].

\subsection{Comparaison entre un gainage précoce et un épistillage-gainage au stade " doigts horizontaux "}

Une expérimentation permettant la comparaison entre un gainage précoce $\left(\mathrm{Sdh}_{-7}\right)$ et un épistillage-gainage au stade "doigts horizontaux " (Sdh) a été réalisée sur une bananeraie de quatrième cycle, située à 250 m d'altitude de la côte au vent, un site où la pression infectieuse observée est élevée.

L'épistillage consiste à éliminer toutes les pièces florales des fruits au champ. Cette opération réalisée au stade "doigts horizontaux " coïncide avec l'abscission des pièces florales. Trois répétitions ont été réalisées en septembre 2000, au cours de trois semaines consécutives de marquage. Chaque semaine, 40 bananiers ont été marqués, et chaque traitement a été ainsi représenté par un ensemble de 20 bananiers complètement dispersés dans la bananeraie expérimentale. Les niveaux de contamination ont été mesurés sur trois fruits prélevés respectivement sur la première, la médiane et la dernière main de chaque régime expérimental récolté 7 semaines après la floraison. 


\subsection{Influence du stade de gainage sur la croissance des fruits}

L'influence du stade de gainage sur la croissance des fruits a été étudiée lors d'une expérimentation comportant cinq traitements : inflorescences gainées aux quatre stades de gainage déjà évoqués et inflorescences non gainées et non épistillées. Les bananiers expérimentaux ont été choisis au stade "fleur pointante" pour être en conformité avec le modèle de croissance des fruits élaboré par Jullien [2]. Trente bananiers par semaine ont été sélectionnés pour expérimenter les cinq traitements, soit six bananiers par traitement. L'étude a été faite au cours de deux semaines consécutives déterminant deux répétitions pour chacun des traitements. La croissance des fruits a été caractérisée par deux paramètres : le nombre moyen de cellules par fruit et le poids moyen d'un fruit pour chaque régime.

\subsubsection{Nombre moyen de cellules par fruit}

Le nombre de cellules moyen par fruit (Ncell) a été évalué à 350 degrés jours après la sortie de l'inflorescence. Ce nombre conditionne la vitesse de remplissage des fruits. Son calcul découle de la mesure de la longueur (L) du doigt médian externe de chaque main de tous les régimes, à la somme de température de 350 degrés jours à partir de la sortie de l'inflorescence [13] : Ncell $=1,16 \times 10^{7} \times \mathrm{L}-1,19 \times 10^{8}$.

Pour chaque régime, Ncell $_{\mathrm{i}}$ a été estimé en calculant le nombre de cellules par fruit à un niveau de main " $\mathrm{i}$ ". Une moyenne pondérée $\left(\right.$ Ncell $\left._{\mathrm{p}}\right)$ a alors été calculée en tenant compte du nombre de fruits par main. Pour ce calcul nous avons considéré uniquement les six premières mains du régime, car l'augmentation de la température sous la gaine n'est pas uniforme et est peu marquée sur les mains du bas [2]. Par ailleurs, cette façon de faire a permis d'homogénéiser les résultats obtenus d'un régime à l'autre, certains n'ayant pas le même nombre de mains et les fruits des dernières mains étant de plus petite taille.
Ainsi :

Ncell $_{\mathrm{p}}=\left\{\left[\left(\mathrm{n}_{1} \times\right.\right.\right.$ Ncell $\left._{1}\right)+\left(\mathrm{n}_{2} \times \mathrm{Ncell}_{2}\right)+$ $\left(n_{3} \times\right.$ Ncell $\left._{3}\right)+\left(n_{4} \times\right.$ Ncell $\left._{4}\right)+\left(n_{5} \times\right.$ Ncell $\left._{5}\right)$

$\left.\left.+\left(\mathrm{n}_{6} \times \mathrm{Ncell}_{6}\right)\right] /\left(\mathrm{n}_{1}+\mathrm{n}_{2}+\mathrm{n}_{3}+\mathrm{n}_{4}+\mathrm{n}_{5}+\mathrm{n}_{6}\right)\right\}$, avec $n_{i}$ nombre de fruits sur la main " $i$ ".

\subsubsection{Poids moyen d'un fruit pour chaque régime}

Le poids moyen d'un fruit pour chaque régime $\left(\mathrm{Pfr}_{\text {moy }}\right)$ a été évalué par le rapport : [poids frais du régime / nombre total de doigts du régime].

Le poids frais des régimes a été mesuré, entièrement, y compris avec le poids de la hampe, à la récolte effectuée au stade 900 degrés jour. Le nombre de mains par régime et le nombre de doigts par main ont été comptés à la floraison pour calculer le nombre de doigts du régime.

\section{7. Évaluation de l'incidence et de la sévérité de la maladie}

Lors de chaque prélèvement, les infections quiescentes des fruits ont été révélées suivant la méthode mise au point par de Lapeyre de Bellaire et al. [14], qui consiste à exposer les fruits à une dose d'éthylène de $1200 \mu \mathrm{L} \cdot \mathrm{L}^{-1}$ pendant 5 jours, dans une enceinte humidifiée, régulée à $32^{\circ} \mathrm{C}$. Le nombre de nécroses par fruit a été comptabilisé à la sortie des fruits.

Le pourcentage de fruits nécrosés (\%Fnéc) par rapport à tous les fruits observés a alors été calculé. Cette variable indique l'incidence de la maladie. La détermination de la moyenne du nombre de nécroses par fruit (Nnéc moy $_{\text {) a permis }}$ d'évaluer la sévérité de la maladie.

\subsection{Analyse statistique des résultats}

Les pourcentages de fruits nécrosés (\%Fnéc) et les moyennes du nombre de nécroses par fruit ( Nnéc $_{\text {moy }}$ ) ont respectivement subi une transformation en $\arcsin \sqrt{\mathrm{x}}$ et en $\sqrt{\mathrm{x}}$ avant leur analyse de variance avec le logiciel Statitcf. En revanche, aucune transformation préalable n'a été nécessaire avant l'analyse de la variance du poids moyen d'un fruit par régime 
Figure 2.

Comparaison des conditions épidémiologiques des expériences réalisées en 2000 et en 1997. Les niveaux de contamination mesurés à la récolte sur des régimes non épistillés et non gainés sont comparés avec la pluviométrie cumulée enregistrée durant les 35 premiers jours de floraison.

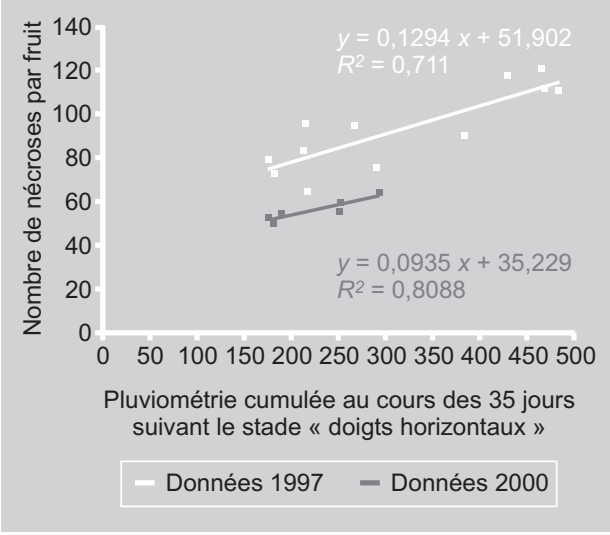

$\left(\mathrm{Pfr}_{\text {moy }}\right)$ et du nombre de cellules des six premières mains des régimes observés $\left(\mathrm{NCell}_{\mathrm{p}}\right)$. La comparaison des moyennes entre les traitements a été réalisée avec le test de Newman-Keuls au seuil de $5 \%$.

\section{Résultats}

\subsection{Influence du stade de gainage sur la contamination des fruits}

Au cours de cette expérimentation, l'incidence de la maladie a été généralement élevée alors que le niveau de contamination des fruits (sévérité) est resté modéré. En moyenne, $81 \%$ des fruits ont présenté au moins une nécrose, et, sur l'ensemble des fruits, il y a eu en moyenne 20 nécroses par fruit. L'analyse de la relation entre la sévérité de la maladie sur les fruits non gainés et la pluviométrie cumulée enregistrée 35 jours après le stade "doigts horizontaux ", période où les sources d'inoculums sont les plus abondantes, révèle que les conditions épidémiologiques de cette expérience ont été moyennement favorables au développement de la maladie (figure 2). En effet, la comparaison des résultats obtenus au cours de cette expérimentation avec ceux d'un essai antérieur réalisé sur la même parcelle [12] montre que les hauteurs de précipitations enregistrés au cours de la période critique n'ont pas été aptes à favoriser un développement très important de la maladie sur les fruits non gainés

Le stade floral auquel la gaine a été posée a eu un effet important à la fois sur l'incidence et sur la sévérité de la maladie (tableau I). Ainsi, quelle que soit la date d'observation des fruits, effectuée soit 7 semaines après la sortie de l'inflorescence, soit à 900 degrés jour, l'incidence et la sévérité de la maladie ont été d'autant plus élevées que le gainage a été réalisé tardivement.

Quel que soit le stade de l'inflorescence au moment du gainage, les fruits non gainés ont toujours été significativement plus contaminés (sévérité) que les fruits gainés.

Tableau I.

Comparaison de l'incidence et de la sévérité de l'anthracnose en fonction des stades de gainage d'inflorescences de bananiers et de la date d'observation des fruits ( 7 semaines après la jetée ou stade récolte à 900 degrés jour).

\begin{tabular}{|c|c|c|c|c|}
\hline \multirow[t]{2}{*}{ Stade de gainage } & \multicolumn{2}{|c|}{$\begin{array}{c}\text { Incidence } \\
\text { (\% fruits infestés) }\end{array}$} & \multicolumn{2}{|c|}{$\begin{array}{c}\text { Sévérité } \\
\text { (nombre de nécroses par fruit) }\end{array}$} \\
\hline & $\begin{array}{l}7^{e} \text { semaine } \\
\text { après la jetée }\end{array}$ & $\begin{array}{l}\text { Stade } \\
\text { récolte }\end{array}$ & $\begin{array}{l}7^{\mathrm{e}} \text { semaine } \\
\text { après la jetée }\end{array}$ & $\begin{array}{l}\text { Stade } \\
\text { récolte }\end{array}$ \\
\hline Stade « doigts horizontaux » $-7 \mathrm{j}$ & $63 \mathrm{a}$ & $80 \mathrm{a}$ & $5 a$ & $8 a$ \\
\hline Stade « doigts horizontaux» & $63 \mathrm{a}$ & $76 \mathrm{a}$ & $6 a b$ & $8 a$ \\
\hline Stade « doigts horizontaux » $+7 \mathrm{j}$ & $77 \mathrm{~b}$ & $85 a b$ & $10 \mathrm{~b}$ & $12 \mathrm{a}$ \\
\hline Stade « doigts horizontaux » $+14 \mathrm{j}$ & $86 \mathrm{c}$ & $90 \mathrm{bc}$ & $22 \mathrm{c}$ & $23 b$ \\
\hline Inflorescence non gainée & $92 \mathrm{c}$ & $96 \mathrm{c}$ & $49 \mathrm{~d}$ & $56 \mathrm{c}$ \\
\hline
\end{tabular}


Les fruits gainés avec un retard de deux semaines $\left(\mathrm{Sdh}_{+14}\right)$ ont été plus sévèrement contaminés que ceux pour lesquels le gainage avait été plus précoce.

L'incidence de la maladie a été aussi plus élevée pour les fruits non gainés ou gainés avec un retard de deux semaines $\left(\mathrm{Sdh}_{+14}\right)$ que pour les fruits ayant subi les autres traitements $\left(\mathrm{Sdh}_{-7}\right.$, $\mathrm{Sdh}$ et $\left.\mathrm{Sdh}_{+7}\right)$, mais il n'y a pas eu de différences significatives entre ces deux traitements [non gainé et $\left.\left(\mathrm{Sdh}_{+14}\right)\right]$. Par ailleurs un gainage en retard d'une semaine $\left(\mathrm{Sdh}_{+7}\right)$ s'est traduit par une augmentation de la sévérité et de l'incidence, même si les différences n'ont pas toujours été significatives par rapport au gainage au stade "doigts horizontaux" (Sdh). Enfin, dans les conditions de cette expérimentation, le gainage précoce ( $\left.\mathrm{Sdh}_{-7}\right)$ n'a pas permis de réduire significativement le niveau de contamination des fruits, par rapport au gainage réalisé au stade " doigts horizontaux" (Sdh).

La corrélation entre le niveau des précipitations durant la période où les sources d'inoculums sont les plus abondantes ( 35 jours après la jetée) et la sévérité de la maladie a été d'autant plus marquée que le gainage des régimes avait été tardif (tableau II). Ainsi, les précipitations durant cette période critique ont peu d'effet sur la sévérité lorsque le gainage est réalisé précocement ou au stade "doigts horizontaux".

\subsection{Comparaison entre un gainage précoce et l'épistillage-gainage au stade " doigts horizontaux"}

L'incidence et la sévérité de la maladie ont été significativement différentes entre les régimes gainés précocement et ceux épistillés et gainés au stade "doigts horizontaux ". La combinaison épistillage/ gainage assurerait donc une meilleure protection des fruits contre la maladie qu'un simple gainage précoce (tableau III). Les deux traitements étudiés ont toutefois permis d'obtenir de faibles niveaux de contamination, puisque moins de 10 nécroses par fruit ont été observés.
Tableau II.

Corrélations existant entre le cumul des précipitations pendant les 35 premiers jours après la jetée de l'inflorescence de bananiers et la sévérité de l'anthracnose mesurée sur les fruits matures. Ces corrélations sont établies pour 6 semaines consécutives de marquage et pour chacun des stades de gainage du régime.

Stade de gainage

Coefficient de corrélation

Stade « doigts horizontaux $»-7 j$

0,43

Stade « doigts horizontaux »

0,57

Stade « doigts horizontaux » +7

0,78

Stade « doigts horizontaux » $+14 \mathrm{j}$

Inflorescence non gainée

0,86

\section{Tableau III.}

Comparaison de l'incidence et de la sévérité de l'anthracnose en fonction des techniques culturales en bananeraie : [gainage précoce au stade « doigts horizontaux » -7 jours] ou [épistillage + gainage au stade “ doigts horizontaux »]. Les mesures ont été réalisées la septième semaine après la floraison.

\begin{tabular}{lcc}
\hline Stade de gainage & $\begin{array}{c}\text { Incidence } \\
\text { (\% fruits infestés) }\end{array}$ & $\begin{array}{c}\text { Sévérité } \\
\text { (nombre de nécroses par fruit) }\end{array}$ \\
\hline Gainage précoce & $87 \mathrm{a}$ & $8 \mathrm{a}$ \\
Épistillage + gainage & $55 \mathrm{~b}$ & $2 \mathrm{~b}$
\end{tabular}

$a, b$ : dans une même colonne, les valeurs suivies de la même lettre ne sont pas différentes au seuil de probabilité de $5 \%$ d'après le test de Newman-Keuls.

\subsection{Influence du stade de gainage sur la croissance des fruits}

\subsubsection{Nombre de cellules par fruit}

Il y a eu un effet significatif du stade de gainage de l'inflorescence sur le nombre de cellules moyen par fruit (tableau IV), qui a été d'autant plus élevé que le gainage avait été précoce. Un gainage tardif s'est traduit par une réduction sensible du nombre de cellules moyen. À l'inverse, un gainage précoce a permis d'augmenter le potentiel de remplissage des fruits.

\subsubsection{Poids moyen des fruits}

Plus le gainage a été tardif, plus le poids moyen des fruits a diminué, même si les différences entre les différents stades de gainage ne se sont pas révélées 


\section{Tableau IV.}

Comparaison du nombre moyen de cellules par fruits à la récolte en fonction du stade de gainage de l'inflorescence de bananiers.

\begin{tabular}{lc}
\hline Stade de gainage & Nombre moyen de cellules par fruit $\left(\times 10^{8}\right)$ \\
\hline Stade « doigts horizontaux »-7 $\mathrm{j}$ & $1,27 \mathrm{a}$ \\
Stade « doigts horizontaux » & $1,23 \mathrm{ab}$ \\
Stade « doigts horizontaux » + $\mathrm{j}$ & $1,14 \mathrm{~b}$ \\
Stade « doigts horizontaux »+14 $\mathrm{j}$ & $1,18 \mathrm{~b}$ \\
Inflorescence non gainée & $1,12 \mathrm{~b}$
\end{tabular}

$a, b$ : dans une même colonne, les valeurs suivies de la même lettre ne sont pas différentes au seuil de probabilité de $5 \%$ d'après le test de Newman-Keuls.

\section{Tableau V.}

Comparaison du poids moyen d'un fruit à la récolte en fonction du stade de gainage de l'inflorescence de bananiers.

\begin{tabular}{lc}
\hline Stade de gainage & Poids moyen d'un fruit \\
& $(\mathrm{g})$ \\
\hline Stade « doigts horizontaux »-7 & $163 \mathrm{a}$ \\
Stade « doigts horizontaux » & $163 \mathrm{a}$ \\
Stade « doigts horizontaux » $+7 \mathrm{j}$ & $152 \mathrm{a}$ \\
Stade « doigts horizontaux » $+14 \mathrm{j}$ & $138 \mathrm{a}$ \\
Inflorescence non gainée & $115 \mathrm{~b}$
\end{tabular}

a, $b$ : les valeurs suivies de la même lettre ne sont pas différentes au seuil de probabilité de $5 \%$ d'après le test de Newman-Keuls.

significatives (tableau $V$ ). Le poids des fruits non gainés a été significativement plus faible que celui des autres traitements.

\section{Discussion}

La meilleure protection des fruits contre l'anthracnose a été assurée par un gainage précoce $\left(\mathrm{Sdh}_{-7}\right)$ ou réalisé au stade " doigts horizontaux" (Sdh). L'efficacité de cette protection a diminué ensuite en fonction de l'ampleur du retard pris pour effectuer cette opération.

Le rôle de la pluie est bien connu dans la propagation des maladies à Colletotrichum [15-17]. Il a fait l'objet de nombreuses discussions en ce qui concerne l'anthracnose de la banane [18-20]. La réduction considérable des niveaux de contamination observée sur les fruits des régimes gainés de façon précoce $\left(\mathrm{Sdh}_{-7}\right)$ ou au stade Sdh constitue une preuve supplémentaire de l'importance de la pluie dans le développement de l'anthracnose de la banane. La gaine forme une barrière physique qui limite la circulation de l'eau de pluie sur les fruits. Dès sa mise en place sur le régime, elle réduit la dissémination des conidies sur les fruits et diminue, par conséquent, leur pollution par l'inoculum provenant des pièces florales (auto-inoculum) ou provenant de l'extérieur du régime (allo-inoculum). Il semble donc logique que l'efficacité de cette barrière s'améliore avec la précocité de sa mise en place. D'après ce raisonnement, les fruits ayant bénéficié d'un gainage précoce devraient être davantage protégés que les fruits des régimes gainés au stade Sdh. Cependant, nos résultats n'ont pas mis en évidence de différences entre ces deux traitements, ce qui pourrait être en partie imputable aux conditions climatiques qui ont été peu favorables au développement de la maladie pendant la durée de notre expérimentation. Par ailleurs, le niveau d'inoculum mesuré par piégeage ou par isolement direct sur les pièces florales [11, 12] n'est pas élevé au stade "doigts horizontaux "; il n'augmente réellement que une semaine après, pour atteindre son maximum (14 à 21) $\mathrm{j}$ après le stade Sdh. Cette constatation pourrait également contribuer à expliquer le peu de différences observées entre un gainage précoce et celui réalisé au stade Sdh.

La suppression des pièces florales, qui constituent la majeure source d'auto-inoculum, permet d'améliorer considérablement l'efficacité du gainage au stade Sdh. Cela n'est malheureusement pas réalisable pour un gainage précoce car, à ce stade de développement des fruits, l'inflorescence demeure fermée. La réduction de la sévérité de la maladie par l'épistillage corrobore l'idée selon laquelle les pièces florales constituent la principale source d'inoculum de Colletotrichum musae [12].

Nos résultats ont montré que le poids des fruits des régimes gainés était supérieur à celui des fruits des régimes non gainés. Le 
poids de la hampe florale ayant été pris en compte dans l'estimation du poids moyen d'un fruit par régime, il a peut-être masqué les différences entre les stades de gainage étudiés. Cependant, malgré de faibles différences, il est apparu un gradient du poids des fruits en fonction de la précocité du stade de gainage. Ce gradient a aussi pu être constaté sur le nombre moyen de cellules par fruit qui pourrait être lié à l'augmentation de la température sous la gaine. En effet, les divisions cellulaires ont lieu au cours des 350 premiers degrés jours qui suivent le stade "fleur pointante" [2]. Ainsi, le poids des fruits augmenterait avec la réalisation précoce de cette opération et une meilleure précision dans l'estimation du poids réel des fruits se serait peut-être traduite par des résultats plus significatifs. Quoiqu'il en soit, notre expérimentation montre qu'il existe un effet bénéfique du gainage sur le développement des fruits [21].

En conclusion, notre travail démontre l'intérêt qui doit être porté au stade de pose de la gaine. Celle-ci doit incontestablement être posée avant le stade "doigts horizontaux " afin non seulement d'assurer une meilleure croissance des fruits et de réduire les dégâts liés au thrips de la rouille argentée, mais aussi de conserver son effet protecteur contre l'anthracnose des bananes [22]. Un gainage en retard d'une ou deux semaines représente un risque important de contamination des fruits, risque qui est d'autant plus important en conditions de forte pluviosité. Le gainage précoce peut être une recommandation intéressante dans les exploitations qui sont souvent en retard dans les travaux de gainage car, dans ces conditions, le retard accumulé sera de toute façon moins préjudiciable que si la recommandation est de gainer au stade " doigts horizontaux ".

L'épistillage au champ, combiné à un gainage au stade "doigts horizontaux ", permet une meilleure protection qu'un gainage précoce. Toutefois, cette technique reste coûteuse en main d'œuvre et, en fonction des conditions de l'exploitation (zone climatique, organisation de la main d'œuvre), l'une ou l'autre de ces techniques pourra être adoptée pour limiter la contamination des fruits au champ dans l'espoir de raisonner la lutte chimique ou de s'en affranchir.

\section{Références}

[1] Ganry J., Recherche d'une méthode d'estimation de la date de récolte du bananier à partir de données climatiques dans les conditions des Antilles, Fruits 33 (1978) 669680.

[2] Jullien A., Croissance, développement et qualité des fruits du bananier (Musa spp. groupe AAA cv. Grande Naine). Modélisation de la répartition des assimilats entre les fruits du régime, Institut national agronomique de Paris-Grignon, Paris, France, thèse, 2000, $92 \mathrm{p}$.

[3] Swinburne T.R., Quiescent infections in post-harvest diseases, in: Dennis C. (Eds.), Post-harvest pathology of fruits and vegetables, Acad. Press, London, UK, 1983, 1-21.

[4] Muirhead I.F., Deverall B.J., Role of appressoria in latent infection of banana fruits by Colletotrichum musae, Physiol. Plant Pathol. 19 (1981) 77-84.

[5] Emmet R.W., Parbery D.G., Appressoria, Annu. Rev. Phytopathol. 23 (1975) 421-454.

[6] Meredith D.S., Studies on Gloeosporium musarum Cke and Massee causing storage rots of Jamaican bananas. I. Anthracnose and its chemical control, Ann. Appl. Biol. 48 (1960) 279-290.

[7] Frossard P., Action du thiabendazole et du benlate sur l'anthracnose des bananes et son champignon pathogène Colletotrichum musae, Fruits 24 (1969) 365-379.

[8] de Lapeyre de Bellaire L., Dubois C., Distribution of thiabendazole-resistant Colletotrichum isolates from Guadeloupe banana plantations, Plant Dis. 81 (1997) 1378-1383.

[9] Dorel M., Lafforgue A., Breteaud P., Le Breton M., Étude de la contamination des eaux de ruissellement par les pesticides utilisés en bananeraie, Rapport d'exécution du projet CORDET 93 DA 14, Cirad-Flhor, Guadeloupe, France, 1996, 31 p.

[10] de Lapeyre de Bellaire L., Bio-écologie de Colletotrichum musae (Berk. \& Curt.) Arx, agent de l'anthracnose des bananes, dans les conditions tropicales humides de la 
Guadeloupe, Univ. Paris-Sud, Orsay, France, thèse, 1999, $100 \mathrm{p}$.

[11] de Lapeyre de Bellaire L., Mourichon X., The pattern of fungal contamination of the banana bunch during its development and potential influence on incidence of crown-rot and anthracnose diseases, Plant Pathol. 46 (1997) 481-489.

[12] de Lapeyre de Bellaire L., Chillet M., Dubois C., Mourichon X., Importance of different sources of inoculum and dispersal methods of conidia of Colletotrichum musae, the causal agent of banana anthracnose, for fruit contamination, Plant Pathol. 49 (2000) 782790.

[13] Jullien A., Munier-Jolain N., Malézieux E., Chillet M., Ney B., Effect of pulp cell number and assimilate availability on dry matter accumulation rate in a banana fruit [Musa sp. AAA group 'Grande Naine' (Cavendish subgroup)], Ann. Bot.-London 88 (2001) 321330.

[14] de Lapeyre de Bellaire L., Chillet M., Mourichon X., Elaboration of an early quantification method of quiescent infections of Colletotrichum musae (Berk. \& Curt.) von Arx on bananas, Plant Dis. 84 (2000) 128-133.

[15] Fitzell R.D., Peak C.M., The epidemiology of anthracnose disease of mango: inoculum sources, spore production and dispersal, Ann. Appl. Biol. 104 (1984) 53-59.

[16] Denham T.G., Waller J.M., Some epidemiological aspects of postbloom fruit drop disease (Colletotrichum gloeosporioides) in citrus, Ann. Appl. Biol. 98 (1981) 65-77.

[17] Waller J.M., Colletotrichum diseases of perennial and other cash crops, in: Bailey J.A. Jeger M.J. (Eds.), Colletotrichum: biology, pathology and control, $\mathrm{CAB}$ international, Wallingford, UK, 1992, pp. 167-185.

[18] Dastur J.F., Spraying for ripe rot of plantain fruit, Agric. J. India 11 (1916) 142-149.

[19] Simmonds J.H., Mitchell R.S., Black end and anthracnose of the banana with special reference to Gloeosporium musarum Cke. and Mass, B. Coun. Sci. Ind. Res. Aust. 131 (1940) 1-63.

[20] Shillingford C.A., Occurrence of banana fruit-rot fungi in Jamaican boxing plants, Plant Dis. Rep. 60 (1976) 788-793.

[21] Ganry J., Influence du gainage des régimes de bananiers avec une housse de polyéthylène sur la température des fruits dans les conditions de Neufchâteau (Guadeloupe), Fruits 30 (1975) 735-738.

[22] Rey F., Rouille argentée des bananes martiniquaises : distribution et méthode de lutte, Fruits 57 (1) (2002) 3-10. 


\section{El embolsado temprano de los racimos de bananos mejora el crecimiento de los frutos y su estado sanitario frente a la antracnosis (Colletotrichum musae).}

Resumen - Introducción. La antracnosis del banano, causada por Colletotrichum musae, es una enfermedad de conservación que suele controlarse mediante tratamientos fungicidas tras la cosecha. Actualmente, se buscan otros medios de lucha para sustituir este control químico; medios como el desflore y embolsado de las inflorescencias efectuado habitualmente en la fase "dedos horizontales" (FDH). Habida cuenta de la precocidad de la contaminación de los frutos por C. musae, del papel desempeñado por el agua de lluvia como agente de dispersión de los conidios y del efecto barrera que tiene la bolsa en esta dispersión, quisimos evaluar las consecuencias de un embolsado temprano o tardío en el nivel de contaminación de los frutos. Por otra parte, quisimos evaluar la posibilidad de sustituir el desflore en campo por un embolsado en una fase muy temprana, más fácil de efectuar. Por último, quisimos verificar que un embolsado temprano podía implicar un mejor llenado de los frutos. Material y métodos. Comparamos diferentes fases de embolsado (FDH-7 d, FDH, FDH+7 d, FDH+14 d, racimos no embolsados) en cuanto a sus efectos en la contaminación de los frutos y, también, en su crecimiento (número de células por fruto, peso medio del fruto). El embolsado temprano (FDH-7) fue también comparado con la combinación desflore/embolsado en la fase FDH que es una técnica eficaz, pero costosa en mano de obra. Resultados. Los índices de contaminación de los frutos fueron significativamente más altos en los racimos embolsados tardíamente (FDH+7, FDH+14) y aún peores en los racimos no embolsados. Los niveles más bajos de contaminación se midieron en frutos embolsados precozmente $(\mathrm{FDH}-7) \mathrm{O} F \mathrm{FH}$, sin que existieran diferencias significativas entre estos dos tratamientos. Por otra parte, el embolsado temprano no redujo tanto la contaminación de las frutas como el desflore en campo asociado al embolsado en la FDH. Por último, la fase de embolsado influyó en el crecimiento de los frutos. Conclusión. Se aconseja la colocación temprana de la bolsa antes de la FDH, o incluso antes (FDH-7).

Francia (Guadalupe) / Musa acuminata / control cultural / antracnosis / Colletotrichum musae / contaminación / etapas de desarrollo de la planta / crecimiento / embolse

To access this journal online: www.edpsciences.org 